\title{
The role of generalizability in moral and political psychology
}

\author{
Elizabeth A. Harris \\ New York University \\ eah561@nyu.edu \\ Philip Pärnamets \\ Karolinska Institutet \\ philip.parnamets@ki.se \\ https://philipparnamets.github.io \\ William J. Brady \\ Yale University \\ william.brady@yale.edu \\ Claire E. Robertson \\ New York University \\ cer493@nyu.edu \\ Jay J. Van Bavel \\ New York University \\ jay.vanbavel@nyu.edu
}

994/1000 words

Conflict of interest declaration: There are no conflicts of interest to report for any of the authors.

Funding statement: This publication was made possible through the support of: John Templeton Foundation (\#61378; PP \& JVB), Swedish Research Council (2016-06793; PP), and the Social Science and Humanities Research Council of Canada (752-2018-0213; EH).

Abstract: The aim of the social and behavioral sciences is to understand human behavior across a wide array of contexts. Our theories often make sweeping claims about human nature, assuming that our ancestors or offspring will be prone to the same biases and preferences. Yet we gloss over the fact that our research is often based in a single temporal context with a limited set of stimuli. Political and moral psychology are domains in which the context and stimuli are likely to matter a great deal (Van Bavel et al., 2016). In response to Yarkoni (see BBS issue) we delve into topics related to political and moral psychology that likely depend on features of the research. These topics include understanding differences between liberals and conservatives, when people are willing to sacrifice someone to save others, the behavior of political leaders, and the dynamics of intergroup conflict. 
The aim of the social and behavioral sciences is to understand human behavior across a wide array of contexts. Our theories often make sweeping claims about human nature, assuming that our ancestors or offspring will be prone to the same biases and preferences. Yet we gloss over the fact that our research is often based in a single temporal context with a limited set of stimuli. Political and moral psychology are domains in which the context and stimuli are likely to matter a great deal (Brandt \& Wagemans, 2017; Van Bavel et al., 2016). Understand differences between liberals and conservatives, when people are willing to sacrifice someone to save others, the behavior of political leaders, and the dynamics of intergroup conflict, all likely depend on features of the research.

One of the major challenges of political psychology is understanding the generalizability of stimuli (see Yarkoni, this issue). Political psychologists often make broad claims about the differences (or lack thereof) between conservatives and liberals. However, the field's findings can vary widely depending on the specific stimuli used (e.g., topics, policies, etc.). Whether American Democrats or Republicans, for example, report higher belief superiority depends on the political issue researchers asked about (Toner et al. 2013). Scientists can therefore select different issues to show, alternatively, that people on the left or right of the political spectrum are more prone to a sense of superiority about their beliefs. It is only when looking across multiple topics that the overall quadratic relationship emerges (Harris \& Van Bavel, 2021), suggesting the people on both ends of the spectrum are similarly prone to feelings of superiority when their attitudes are extreme.

This issue of non-generalizable stimuli extends to research on moral psychology as well. For instance, the victims of harm are often described using vague terminology, such as five nondescript workers on a track about to be run over by a trolley. In many studies, the victims are genderless, raceless, etc. (Hester \& Gray, 2020), which makes it difficult to generalize the findings to more vivid or real-world judgments (FeldmanHall et al., 2012). Yet the research suggests that moral judgements are heavily influenced by contextual information and stimuli.

Another often-overlooked aspect of generalizability in both political and moral psychology is the changing temporal dynamics. Historical evidence demonstrates that core concepts studied by political psychologists, such as partisanship and polarization, are changing across time (Bartels 1996; Abramowitz \& Webster 2016; Baldassarri \& Gelman 2008; Kozlowsky \& Murphy, 2020). Furthermore, the stimuli used to study these phenomena often include specific policies or politicians who are well known at a given time and elicit a host of associations related to that period of time.

Temporal factors occurring "outside the lab" may also influence the interactions between moral and political psychology. For instance, 'political elites' in the United States use more moral language when their party is not in power (Wang \& Inbar, 2020), demonstrating the changing nature of moral language usage due to external (and typically unacknowledged) factors 
(see Brady et al., 2019). Similarly, moral language changes over time (cf. Wheeler, McGrath \& Haslam, 2019). The question of temporal generalizability is a foundational question of the historical versus lawlike nature of psychological findings (Gergen, 1973). A critical next step in psychological research must be examining whether trends related to political or moral reasoning are robust across time.

It is also important to avoid the assumption that reactions to political and moral stimuli in one cultural context reveal insights into individuals in another cultural context. For example, claims about growing rates of political polarization are often made based on U.S. samples, with participants embedded within a two-party political system (Finkel et al., 2020). Recent evidence suggests that other countries show varying trajectories of political polarization likely based on context-specific characteristics of their political system (Boxell, Gentzkow \& Shapiro, 2020). Future work should include international samples or else constrain their claims to reflect the current sample.

In moral psychology, foundational research on moral intuitions largely ignored the prospect of cultural variation. For example, dual-processing theories developed by studying trolley problem dilemmas exclusively used evidence from WEIRD samples (Greene et al., 2001), yet recent cross-cultural work demonstrates that participants' intuitions about trolly problem dilemmas are notably different in eastern cultures (Rehman \& Dzionek-Kozłowska, 2020) and non-industrialized cultures (Sorokowski et al. 2020). Moral and political psychologists should be aware of the type of evidence required to make claims of strong or weak universality (Norenzayan \& Heine, 2005), and make explicit reference to cultural generalizability when drawing broad conclusions from a small number of studies or restricted samples (Bago et al., 2021).

Moral and political psychology face major generalizability challenges with regard to the content of research materials, temporal variability, and cultural differences. To address these challenges, part of the solution involves methodological and analytic changes (see Yarkoni, this issue). While important, these changes alone cannot fully address questions of generalizability. Here we join many recent observers in calling for better theory development and employment of formal tools to make more precise theoretical claims (Muthukrishna \& Henrich, 2019; Guest \& Martin, 2020; van Rooij \& Baggio, 2021). Improved theory will not only help close the distance between theoretical claims and empirical tests, but guide and facilitate interdisciplinary research. The latter may be particularly important in moral and political psychology, as its subject matter clearly intersects with explanatory efforts in many adjacent disciplines.

One hope is that, with changes to our empirical and theoretical practices, future researchers will be better placed to assess the generalizability of research in moral and political psychology. One potential future for this work is for researchers to organize themselves not only 
in cross-disciplinary and cross-cultural consortia but also cross-temporally. This would mean planning for research projects to investigate fundamental questions over longer time periods that span entire grant cycles or even individual careers. Conducting research with more diverse samples of participants and stimuli, across social and political contexts, will provide an exciting foundation for the future of the field. 


\section{References}

Abramowitz, A. I., \& Webster, S. (2016). The rise of negative partisanship and the nationalization of US elections in the 21st century. Electoral Studies, 41, 12-22.

Bago, B., Aczel, B., Kekecs, Z., Protzko, J., Kovacs, M., Nagy, T., ... Dutra, N. B. (2021). Moral thinking across the world: Exploring the influence of personal force and intention in moral dilemma judgments. https://doi.org/10.31234/osf.io/9uaqm

Baldassarri, D., \& Gelman, A. (2008). Partisans without constraint: Political polarization and trends in American public opinion. American Journal of Sociology, 114(2), 408-446.

Bartels, L. M. (2000). Partisanship and voting behavior, 1952-1996. American Journal of Political Science, 35-50.

Boxell, L., Gentzkow, M., \& Shapiro, J. M. (2020). Cross-country trends in affective polarization (No. w26669). National Bureau of Economic Research.

Brady, W. J., Wills, J. A., Burkart, D., Jost, J. T., \& Van Bavel, J. J. (2019). An ideological asymmetry in the diffusion of moralized content on social media among political leaders. Journal of Experimental Psychology: General, 148(10), 1802.

Brandt, M. J., \& Wagemans, F. (2017). From the political here and now to generalizable knowledge. Translational Issues in Psychological Science, 3(3), 317.

FeldmanHall, O., Mobbs, D., Evans, D., Hiscox, L., Navrady, L., \& Dalgleish, T. (2012). What we say and what we do: The relationship between real and hypothetical moral choices.

Cognition, 123(3), 434-441.

Finkel, E. J., Bail, C. A., Cikara, M., Ditto, P. H., IIyengar, S., Klar, S., Mason, L., McGrath, M. C., Nyhan, B., Rand, D., Skitka, L., Tucker, J. A., Van Bavel, J.J., Wang, C. S. \& Druckman, J. N. (2020). Political sectarianism in America: A poisonous cocktail of othering, aversion, and moralization. Science, 370, 533-536.

Gergen, K. J. (1973). Social psychology as history. Journal of Personality and Social psychology, 26(2), 309.

Greene, J. D., Sommerville, R. B., Nystrom, L. E., Darley, J. M., \& Cohen, J. D. (2001). An fMRI investigation of emotional engagement in moral judgment. Science, 293(5537), 2105-2108. 
Guest, O., \& Martin, A. E. (2020). How computational modeling can force theory building in psychological science. https://doi.org/10.31234/osf.io/rybh9

Harris, E. A., \& Van Bavel, J. J. (2021). Preregistered Replication of "Feeling Superior Is a Bipartisan Issue: Extremity (Not Direction) of Political Views Predicts Perceived Belief Superiority." Psychological Science. https://doi.org/10.1177/0956797620968792

Hester, N., \& Gray, K. (2020). The moral psychology of raceless, genderless strangers. Perspectives on Psychological Science, 15(2), 216-230.

Kozlowski, A. C., \& Murphy, J. P. (2020). Issue alignment and partisanship in the American public: Revisiting the 'partisans without constraint'thesis. Social Science Research, 102498.

Muthukrishna, M., \& Henrich, J. (2019). A problem in theory. Nature Human Behaviour, 3(3), 221-229.

Norenzayan, A., \& Heine, S. J. (2005). Psychological universals: What are they and how can we know?. Psychological bulletin, 131(5), 763-784.

Rehman, S., \& Dzionek-Kozłowska, J. (2020). The Chinese and American Students and the Trolley Problem: A Cross-cultural Study. Journal of Intercultural Communication, 20(2), 31-41.

Sorokowski, P., Marczak, M., Misiak, M., \& Białek, M. (2020). Trolley Dilemma in Papua. Yali horticulturalists refuse to pull the lever. Psychonomic bulletin \& review, 1-6.

Toner, K., Leary, M. R., Asher, M. W., \& Jongman-Sereno, K. P. (2013). Feeling superior is a bipartisan issue: Extremity (not direction) of political views predicts perceived belief superiority. Psychological Science, 24(12), 2454-2462.

Van Bavel, J. J., Mende-Siedlecki, P., Brady, W. J., \& Reinero, D. A. (2016). Contextual sensitivity in scientific reproducibility. Proceedings of the National Academy of Sciences, 113(23), 6454-6459.

van Rooij, I., \& Baggio, G. (2021). Theory before the test: How to build high-verisimilitude explanatory theories in psychological science. Perspectives on Psychological Science, 1745691620970604.

Wang, S. Y. N., \& Inbar, Y. (2020). Moral-Language Use by US Political Elites. Psychological Science, 0956797620960397. 
Wheeler, M. A., McGrath, M. J., \& Haslam, N. (2019). Twentieth century morality: The rise and fall of moral concepts from 1900 to 2007. PLoS one, 14(2), e0212267. 